\title{
ON AN INVERSE PROBLEM FOR THE SYSTEM OF DIRAC EQUATIONS WITH DISCONTINUITY CONDITIONS ON THE WHOLE AXIS
}

\author{
GULSHAN M. AZIMOVA AND HIDAYAT M. HUSEYNOV
}

\begin{abstract}
The Dirac operator on the whole axis with conditions of discontinuity at some point is considered. Basic equations of the inverse problem are introduced. The uniqueness theorem of the solution of the inverse problem is proved.
\end{abstract}

\section{Introduction}

Let us consider the system of Dirac equations

$$
B y^{\prime}+\Omega(x) y=\lambda y, \quad-\infty<x<+\infty
$$

with discontinuity conditions at some point $a \in(-\infty,+\infty)$

$$
\begin{aligned}
& y_{1}(a-0)=y_{1}(a+0), \\
& y_{2}(a-0)=\alpha y_{2}(a+0) .
\end{aligned}
$$

Here

$$
B=\left(\begin{array}{cc}
0 & 1 \\
-1 & 0
\end{array}\right), \quad \Omega(x)=\left(\begin{array}{cc}
p(x) & q(x) \\
q(x) & -p(x)
\end{array}\right), \quad y=\left(\begin{array}{l}
y_{1} \\
y_{2}
\end{array}\right)
$$

$\alpha$ is a real number, $0<\alpha \neq 1, p(x), q(x)$ are real-valued functions satisfying the condition

$$
\int_{-\infty}^{\infty}\{|p(x)|+|q(x)|\} d x<+\infty .
$$

In the present paper we study direct and inverse problems for the equation (1.1) with the conditions (1.2). In the absence of the discontinuity condition, i.e. when $\alpha=1$ in the condition (1.2), various variants of inverse problems were studied in [4-9].

Similar problems for the equation (1.1) with discontinuity conditions were considered in $[2,3,10,11]$.

2010 Mathematics Subject Classification. 34L25, 34L40, 47A40.

Key words and phrases. inverse scattering problems, system of Dirac equations, discontinuity conditions, scattering function, Jost solution. 
Note that problem (1.1)-(1.2) can be written in the form of the system of first order differential equations with discontinuous coefficients

$$
\left\{\begin{array}{l}
\frac{1}{\rho(x)}\left(\rho(x) y_{2}\right)^{\prime}+p(x) y_{1}+q(x) y_{2}=\lambda y_{1} \\
-y_{1}^{\prime}+q(x) y_{1}-p(x) y_{2}=\lambda y_{2}
\end{array}\right.
$$

where the function $\rho(x)=\alpha$ for $x>a$ and $\rho(x)=1$ for $x<a$

\section{The direct scattering problem}

We will call the functions $e^{ \pm}(x, \lambda)$ satisfying the equation (1.1), the discontinuity condition (1.2) and the condition at the infinity

$$
\lim _{x \rightarrow \pm \infty} e^{ \pm}(x, \lambda) e^{\mp i \lambda x}=\left(\begin{array}{c}
1 \\
\mp i
\end{array}\right)
$$

the Jost solutions.

It is easy to show that if $p(x)=q(x)=0$, then the functions

$$
e_{0}^{ \pm}(x, \lambda)=\left\{\begin{array}{l}
\left(\begin{array}{c}
1 \\
\mp i
\end{array}\right) e^{ \pm i \lambda x}, \quad \pm x> \pm a \\
\frac{1+\alpha^{ \pm 1}}{2}\left(\begin{array}{c}
1 \\
\mp i
\end{array}\right) e^{ \pm i \lambda x}+\frac{1-\alpha^{ \pm 1}}{2}\left(\begin{array}{c}
1 \\
\pm i
\end{array}\right) e^{ \pm i \lambda(2 a-x)}, \pm x< \pm a
\end{array} .\right.
$$

are the Jost solutions. Under the condition (1.3) problem (1.1)-(1.2) for all $\lambda \in$ $(-\infty,+\infty)$ has the Jost solutions $e_{0}^{ \pm}(x, \lambda)$ representable in the form

$$
e^{ \pm}(x, \lambda)=e_{0}^{ \pm}(x, \lambda) \pm \int_{x}^{ \pm \infty} K^{ \pm}(x, t)\left(\begin{array}{c}
1 \\
\mp i
\end{array}\right) e^{ \pm i \lambda t} d t
$$

where the kernels $K^{ \pm}(x, t)$ satisfy the inequalities

$$
\pm \int_{x}^{ \pm \infty}\left\|K^{ \pm}(x, t)\right\| d t \leq e^{c \sigma^{ \pm(x)}}-1
$$

where $c$ is some positive constant,

$$
\sigma^{ \pm}(x)= \pm \int_{x}^{ \pm \infty}\|\Omega(t)\| d t
$$

$\|\cdot\|$ is an operator norm in the Euclidean space $C^{2}$.

Furthermore, the following relations are fulfilled:

$$
\begin{array}{r}
\lim _{t \rightarrow \pm 0} \int_{a}^{+\infty}\left\|B K^{ \pm}(x, x+t)-K^{ \pm}(x, x+t) B \mp \Omega(x)\right\| d x=0, \\
\lim _{t \rightarrow \pm 0} \int_{\infty}^{a}\left\|B K^{ \pm}(x, x+t)-K^{ \pm}(x, x+t) B \mp \frac{1+\alpha^{ \pm 1}}{4} \Omega(x)\right\| d x=0 .
\end{array}
$$

Note that the operator generated by the left hand side of the equation (1.4) is a self-adjoint operator in the space $L_{2, \rho}\left(-\infty,+\infty ; C^{2}\right)$. The spectrum of this operator is purely continuous and fills the whole real axis. 
For all real values of $\lambda$ the pairs of functions $e^{+}(x, \lambda), \overline{e^{+}(x, \lambda)}$ and $e^{-}(x, \lambda)$, $\overline{e^{-}(x, \lambda)}$ form fundamental systems of the solutions of problem (1.1)-(1.2). Hence we easily obtain that the problem (1.1)-(1.2) for all real $\lambda$ has the solutions

$$
\begin{aligned}
& u^{+}(x, \lambda)=t(\lambda) e^{-}(x, \lambda)=\overline{e^{+}(x, \lambda)}+r^{+}(\lambda) e^{+}(x, \lambda), \\
& u^{-}(x, \lambda)=t(\lambda) e^{+}(x, \lambda)=\overline{e^{-}(x, \lambda)}+r^{-}(\lambda) e^{-}(x, \lambda),
\end{aligned}
$$

where

$$
\begin{gathered}
r^{+}(\lambda)=-\frac{\overline{b(\lambda)}}{a(\lambda)}, \quad r^{-}(\lambda)=\frac{b(\lambda)}{a(\lambda)}, \quad t(\lambda)=\frac{1}{a(\lambda)}, \\
a(\lambda)=\frac{W\left[e^{+}(x, \lambda), e^{-}(x, \lambda)\right]}{2 i}, \quad b(\lambda)=-\frac{W\left[e^{+}(x, \lambda), \overline{e^{-}(x, \lambda)}\right]}{2 i},
\end{gathered}
$$

while $W[y, z]=\widetilde{y}(x) B z(x) \rho(x)$ is Wronskian of two solutions of problems (1.1)(1.2) independent of $x$ in the intervals $(-\infty, a),(+\infty, a)$ and

$$
\left.W[y ; z]\right|_{x=a-0}=\left.W[y ; z]\right|_{x=a+0} .
$$

These solutions are said to be eigen-functions of the left $\left(u^{-}(x, \lambda)\right)$ and the right $\left(u^{+}(x, \lambda)\right)$ scattering problems, the coefficients $r^{-}(\lambda), r^{+}(\lambda)$ and $t(\lambda)$ are called left and right reflection coefficients and transmission coefficient:

It is easy to show that in the case $\Omega(x) \equiv 0$ we have

$$
\begin{gathered}
a_{0}(\lambda)=\frac{W\left[e_{0}^{+}(x, \lambda), e_{0}^{-}(x, \lambda)\right]}{2 i}=\frac{1+\alpha}{2 i}, \\
b_{0}(\lambda)=-\frac{W\left[e_{0}^{+}(x, \lambda), \overline{e_{0}^{-}(x, \lambda)}\right]}{2 i}=\frac{1-\alpha}{2 i} e^{2 i \lambda a}, \\
r_{0}^{+}(\lambda)=-\frac{1-\alpha}{1+\alpha} e^{-2 i \lambda a}, \quad r_{0}^{-}(\lambda)=\frac{1-\alpha}{1+\alpha} e^{2 i \lambda a} .
\end{gathered}
$$

From the representation $(2.3)$ of the solutions $e_{0}^{+}(x, \lambda)$ and from formula $(2.5)$ we have the following lemma.

Lemma 2.1. The functions $a(\lambda)$ and $b(\lambda)$ have the following properties

1)

$$
a(\lambda)=a_{0}(\lambda)+\int_{0}^{\infty} \varphi(t) e^{i \lambda t} d t
$$

2)

$$
b(\lambda)=b_{0}(\lambda)+\int_{-\infty}^{\infty} \psi(t) e^{i \lambda t} d t,
$$

where $\varphi(t) \in L_{1}(0, \infty), \psi(t) \in L_{1}(-\infty,+\infty)$,

3) $|a(\lambda)|^{2}-|b(\lambda)|^{2}=\alpha$

4) The function $a(\lambda)$ is regular for $\operatorname{Im} \lambda>0$ and it does not have zeros in the half-plane $\operatorname{Im} \lambda \geq 0$. 


\section{The inverse scattering problem}

The inverse problem of scattering theory for the problem (1.1)-(1.2) consists of determination of the potential $\Omega(x)$ by the left and right scattering data, i.e. by the functions $r^{-}(\lambda)$ or $r^{+}(\lambda)$. It turns at that these functions are related to each other, exactly it is easy to see that the following relations hold

$$
r^{-}(\lambda)=-\overline{r^{+}(\lambda)} \frac{\overline{a(\lambda)}}{a(\lambda)}
$$

moreover,

$$
a(z)=\frac{1+\alpha}{2} \exp \left\{-\frac{1}{2 \pi i} \int_{-\infty}^{\infty} \frac{\ln \left[\left(1-\left|r^{+}(\lambda)\right|^{2}\right) \frac{(\alpha+1)}{4 \alpha}^{2}\right]}{\lambda-z} d \lambda\right\}
$$

Thus, it suffices to determine the potential by $r^{+}(\lambda)$.

Further, according to the definition of the function $r^{ \pm}(\lambda)$ and lemma 2.1, these functions are continuous on $R$ and

$$
\begin{gathered}
\left|r^{ \pm}(\lambda)\right|<1, \quad \lambda \in R, \\
r^{ \pm}(\lambda)-r_{0}^{ \pm}(\lambda)=\int_{-\infty}^{\infty} \varphi^{ \pm}(t) e^{-i \lambda t} d x,
\end{gathered}
$$

where

We have

$$
r_{0}^{ \pm}(\lambda)=\mp \frac{\alpha-1}{\alpha+1} e^{\mp 2 i \lambda a}, \quad \varphi^{ \pm}(x) \in L_{1}(-\infty,+\infty)
$$

Theorem 3.1. For every $x \neq a$ the kernels $K^{ \pm}(x, y)$ from the representations (2.3) satisfy the integral equation

$$
\begin{gathered}
R_{1}^{ \pm}(x, y)+K^{ \pm}(x, y) \pm K^{ \pm}(x, 2 a-y) \frac{\alpha-1}{\alpha+1}\left(\begin{array}{cc}
1 & 0 \\
0 & -1
\end{array}\right) \pm \\
\quad \pm \int_{x}^{ \pm \infty} K^{ \pm}(x, t) R^{ \pm}(t+y) d t=0, \quad \pm y> \pm x,
\end{gathered}
$$

where

$$
\begin{gathered}
R_{1}^{ \pm}(x+y)=\left\{\begin{array}{c}
R^{ \pm}(x+y), \quad \pm x> \pm a \\
\frac{1+\alpha^{ \pm 1}}{2} R^{ \pm}(x+y)+\frac{1-\alpha^{ \pm 1}}{2} R^{ \pm}(2 a-x+y), \pm x< \pm a,
\end{array}\right. \\
R^{ \pm}(x)=\frac{1}{2 \pi} \operatorname{Re} \int_{-\infty}^{+\infty}\left[r^{ \pm}(\lambda)-r_{0}^{ \pm}(\lambda)\right]\left(\begin{array}{cc}
1 & \mp i \\
\mp i & -1
\end{array}\right) e^{ \pm i \lambda x} d \lambda
\end{gathered}
$$

Integral equations (3.3) are called the main Marchenko integral equations of the problem (1.1)-(1.2). These integral equations give an algorithm for finding the potential $\Omega(x)$ by the right reflection coefficient $r^{+}(\lambda)$.

Indeed, by $r^{+}(\lambda)$ we find also $r^{-}(\lambda)$, and on them, according to (3.4) construct the functions $R_{1}^{ \pm}(x, y), R^{ \pm}(x)$ and solving the integral equation (3.3), find the function $R^{ \pm}(x, y)$, and consequently, by means of relations (2.4) determine the potential $\Omega(x)$. 
The following theorem shows that the potential $\Omega(x)$ determined in such way is unique (see $[1,3,11,12]$ ).

Theorem 3.2. Let the conditions (3.1), (3.2) be fulfilled and the functions $R^{ \pm}(x, y)$ be continuous and for all $x^{\prime}>-\infty$

$$
\pm \int_{x^{\prime}}^{ \pm \infty}\left|R^{ \pm}(x)\right| d x<+\infty .
$$

Then, for every fixed $x$ the equation (3.3) has a unique solution $K^{+}(x, \cdot), K^{-}(x, \cdot)$ with the components from $L_{1}(x,+\infty), L(-\infty, x)$ respectively.

\section{Acknowledgements}

This work was supported by the Science Development Foundation under the President of the Republic of Azerbaijan (Grant No EIF/MQM/Elmtehsil-1-201$1(26)-71 / 05 / 1)$.

\section{References}

[1] Z.S. Agranovch, V.A. Marchenko, The inverse problem of scattering theory (in Russian), Kharkov, 1960, 268 pp.

[2] R.Kh. Amirov, On system of differential Dirac equations with conditions of discontinuity of the interval. Ukranian Math. J., 6 (2005), 601-613.

[3] G.M. Azimova, H.M. Huseynov, An inverse scattering problem for system of Dirac equations on the whole axis with conditions of discontinuity at some point. Trans of NAS. Azerb. Issue Math., 36 (2016), no.4, 54-62.

[4] I.S. Frolov, An inverse problem of equations on the whole axis (in Russian), Dokl. Akad. Nauk SSSR, 207 (1972), no.1, 44-47.

[5] M.G. Gasymov, An inverse problem of scattering theory for a system of Dirac equations of order 2n, Trudy Moskov. Mat. Obsh., 19 (1968), 41-112.

[6] M.G. Gasymov, T.T. Dzabiev, Solution of the inverse problem by two spectra for the Dirac equation on a finite interval (in Russian), Dokl. Akad. Nauk SSSR, 22 (1966) no.7, 3-6.

[7] M.G. Gasymov, B.M. Levitan, Determination of the Dirac system from scattering phase (in Russian), Dokl. Akad. Nauk SSSR, 167 (1966), no.6, 1219-1222.

[8] M.G. Gasymov, B.M. Levitan, The inverse problem for a Dirac system (in Russian), Dokl. Akad. Nauk SSSR, 167 (1966), 967-970.

[9] H.M. Huseynov, About Levinson type formula for a system of Dirac equations, $I z v$. Akad. Nauk. Az. SSSR, (1978), no.2, 31-36.

[10] H.M. Huseynov, A.D. Latifova, The main equation for the system of Dirac equations with discontinuity conditions interior to interval (in Russian), Trans. Nat. Acad. Sci. Azerb., 28 (2008), no.1, 63-76.

[11] H.M. Huseynov, An inverse scattering problem for a system of Dirac equations with discontinuity conditions, Proc. Inst. Math. Mech. Natl. Acad. Sci. Azerb., 40 (2014), 215-225.

[12] V.A. Marchenko, Sturm-Liouville operators and their applications (in Russian), Naumkova Dumka Kiev, 1977, 232 pp. 
Gulshan M. Azimova

Azerbaijan State Oil and Industry University, Baku, Azerbaijan

E-mail address: gulshen15@outlook.com

Hidayat M. Huseynov

Baku State University, Baku, Azerbaijan

Institute of Mathematics and Mechanics of NAS of Azerbaijan, Baku, Azerbaijan

E-mail address: hmhuseynov@gmail.com

Received: June 24, 2021; Revised: November 1, 2021; Accepted: November 3, 2021 